\title{
Prediagnostic serum glucose and lipids in relation to survival in breast cancer patients: a competing risk analysis
}

Wahyu Wulaningsih ${ }^{1 *+}$, Mariam Vahdaninia ${ }^{1+}$, Mark Rowley ${ }^{2}$, Lars Holmberg ${ }^{1,3,4}$, Hans Garmo ${ }^{1,4}$, Håkan Malmstrom ${ }^{5}$ Mats Lambe ${ }^{4,6}$, Niklas Hammar ${ }^{5,7}$, Göran Walldius ${ }^{8}$, Ingmar Jungner ${ }^{9}$, Anthonius C. Coolen ${ }^{2}$ and Mieke Van Hemelrijck ${ }^{1,5}$

\begin{abstract}
Background: Abnormal glucose and lipids levels may impact survival after breast cancer (BC) diagnosis, but their association to other causes of mortality such as cardiovascular (CV) disease may result in a competing risk problem.

Methods: We assessed serum glucose, triglycerides (TG) and total cholesterol (TC) measured prospectively 3 months to 3 years before diagnosis in 1798 Swedish women diagnosed with any type of BC between 1985 and 1999. In addition to using Cox regression, we employed latent class proportional hazards models to capture any heterogeneity of associations between these markers and BC death. The latter method was extended to include the primary outcome (BC death) and competing outcomes (CV death and death from other causes), allowing latent class-specific hazard estimation for cause-specific deaths.

Results: A lack of association between prediagnostic glucose, TG or TC with BC death was observed with Cox regression. With latent class proportional hazards model, two latent classes (Class I and II) were suggested. Class I, comprising the majority (81.5\%) of BC patients, had an increased risk of BC death following higher TG levels (HR: 1.87, $95 \%$ Cl: 1.01-3.45 for every log TG increase). Lower overall survival was observed in Class II, but no association for BC death was found. On the other hand, TC positively corresponded to CV death in Class II, and similarly, glucose to death from other causes.
\end{abstract}

Conclusion: Addressing cohort heterogeneity in relation to $B C$ survival is important in understanding the relationship between metabolic markers and cause-specific death in presence of competing outcomes.

Keywords: Breast cancer, Glucose, Lipid, Competing risk, Survival, Latent class

\section{Background}

Disorders in glucose and lipid metabolism have been suggested as a mechanism linking obesity and breast cancer $(\mathrm{BC})[1,2]$. In addition to their roles in carcinogenesis, increasing evidence suggests that abnormal levels of serum glucose and lipids impact survival in $\mathrm{BC}$ patients [3-5]. Most of these studies investigated all-

\footnotetext{
* Correspondence: wahyu.wulaningsih@kcl.ac.uk

${ }^{\dagger}$ Equal contributors

'Cancer Epidemiology Group, Division of Cancer Studies, King's College London, London, UK

Full list of author information is available at the end of the article
}

cause mortality as the outcome of interest. When BCspecific death is studied as the primary outcome, information on other causes of death such as cardiovascular (CV) disease is rarely addressed in the analysis [4]. Given the high survivorship of $\mathrm{BC}[6,7]$ and how glucose and lipids are linked to $\mathrm{CV}$ mortality $[8,9]$, one must consider the possibility of competing risks. For instance, a competing risk situation arises when a person has a common risk factor of dying from both $\mathrm{BC}$ and $\mathrm{CV}$ disease (and other causes), so that any earlier outcome will 'prevent' the individual from developing others [10]. Interpreting survival data thus becomes difficult because commonly used methods, i.e., Kaplan-Meier survival estimates and Cox' proportional hazards, rely on the
() Biomed Central

(C) 2015 Wulaningsih et al. Open Access This article is distributed under the terms of the Creative Commons Attribution 4.0 International License (http://creativecommons.org/licenses/by/4.0/), which permits unrestricted use, distribution, and reproduction in any medium, provided you give appropriate credit to the original author(s) and the source, provide a link to the Creative Commons license, and indicate if changes were made. The Creative Commons Public Domain Dedication waiver (http://creativecommons.org/publicdomain/zero/1.0/) applies to the data made available in this article, unless otherwise stated. 
assumption of non-informative censoring. When this assumption is met, any censoring due to non-primary events does not affect one's risk of developing the primary outcome, thus such a risk is proportional to the levels of risk factors or covariates observed. However, when competing risks are an issue a heterogeneous association between covariates and the primary outcome may exist, reflecting subpopulations or classes with different mortality risk profiles. This heterogeneity within a cohort is scarcely studied in the context of cancer survival.

The objectives of the present study were to investigate how prediagnostic serum glucose, triglycerides (TG) and total cholesterol (TC) are associated to BC death, and to capture heterogeneity of associations between these markers and BC death which may indicate a competing risk situation. We used prospectively collected data from the Apolipoprotein Mortality Risk (AMORIS) Study and utilised 1) Cox proportional hazards model to assess the link between serum glucose, TG and TC with BC death, and 2) latent class proportional hazards models with $B C$ death as the primary outcome and deaths from $\mathrm{CV}$ disease and other causes as non-primary outcomes to capture heterogeneity of $\mathrm{BC}$ mortality risk.

\section{Methods}

\section{Study population}

The Apolipoprotein Mortality Risk (AMORIS) Study has been described in detail elsewhere $[11,12]$. Briefly, the recently updated AMORIS database comprises 812,073 individuals with blood samples sent for laboratory testing to the Central Automation Laboratory (CALAB) in Stockholm, Sweden. Individuals recruited were mainly from the greater Stockholm area, and either healthy and having laboratory testing as a part of general check-up, or outpatients referred for laboratory testing. None of the participants were inpatients at the time the samples were analysed. In the AMORIS study, the CALAB database was linked to Swedish national registries such as the Swedish National Cancer Register, the Hospital Discharge Register, the Cause of Death Register, the consecutive Swedish Censuses during 1970-1990, and the National Register of Emigration using the Swedish 10digit personal identity number, providing complete follow-up information until 31 December 2011.

From the AMORIS population, we selected 1798 women with an incident diagnosis of BC between 1985 and 1999 who had baseline measurements of serum glucose, TG and TC within 3 months to 3 years prior to diagnosis. Diagnosis of BC was obtained from the Swedish National Cancer Register using the Seventh Revision of the International Classification of Diseases code (ICD7 code: 174), and information on cause-specific deaths (BC death, CV death) was obtained from the Swedish Cause of Death Register. Follow-up time was defined as the time from diagnosis until death from any causes, emigration, or end of study (31 December 2011), whichever occurred first. The ethics review board of the Karolinska Institute approved the study, and permits were obtained from Swedish Data Inspection to correlate laboratory results with Swedish national registers. Anonymity of participants was maintained throughout the study. Participant informed consent was not required for this register linkage study [13].

\section{Serum glucose and lipids measurements}

Serum levels of glucose $(\mathrm{mmol} / \mathrm{L})$, TG $(\mathrm{mmol} / \mathrm{L})$, and TC $(\mathrm{mmol} / \mathrm{L})$ were measured enzymatically with standard methods [12]. All three markers were measured at the same day, within 3 months to 3 years prior to diagnosis. This timeframe was selected to capture metabolic derangements during ongoing malignancy process while excluding effects of breast cancer diagnostic or treatment interventions. All measurements were fully automated with automatic calibration and performed at one accredited laboratory [11]. TG levels were not normally distributed, and therefore we used log-transformed values of all markers in addition to their quartiles in the analysis.

\section{Covariates}

Information on fasting status at baseline measurements (fasting, non-fasting, unknown) was obtained from the CALAB database. Socioeconomic status (SES; white collar, blue collar, unemployed or unknown) was based on occupational groups in the Population and Housing Census and classified all gainfully employed subjects as manual workers and non-manual workers, which were referred to as blue collar and white collar workers, respectively [14].

\section{Statistical analysis}

We began by employing multivariable Cox proportional hazards regression to assess the association between logtransformed values and quartiles of glucose, TG and TC and the risk of $\mathrm{BC}$ death as the primary outcome, $\mathrm{CV}$ death and other death as competing outcomes. Adjustment was performed for potential confounders including age at diagnosis, SES, and fasting status at baseline measurements. Glucose, TG and TC were each analysed while adjusting for the other two markers as continuous variables. The proportionality of hazards assumption was met after assessing time-varying covariates which were the cross-products of each variable and time. To assess any potential competing risk, we used cumulative incidence functions to display the proportions of deaths from $\mathrm{BC}, \mathrm{CV}$ disease and other causes by quartiles of glucose, TG, and TC. 
We further investigated the association between serum glucose, TG and TC and $\mathrm{BC}$ survival using a latent class proportional hazards model. Latent class analysis has been used to identify different classes or latent variables within a given population which underlies the pattern of association between observed covariates [15]. In medical research, the latent class variable has been incorporated into various regression analyses, including Cox proportional hazards models, to allow identification of subgroups with different risk profiles [16-18]. To capture heterogeneity in the context of $\mathrm{BC}$ survival, we extended the proportional hazards model to encompass the latent class variable in addition to glucose, TG and TC, which were assessed as continuous variables. The number of latent classes present in the cohort was identified with Bayesian model selection. To assess BC-specific death whilst accounting for competing risks, we incorporated $\mathrm{BC}$ death as the primary outcome and deaths from $\mathrm{CV}$ disease and other causes as non-primary outcomes into the latent class proportional hazards model. Class membership probabilities were retrospectively predicted based on associations between covariates and events. Independent samples $\mathrm{T}$-test and $\mathrm{Chi}^{2}$ test were used to assess differences in characteristics of study participants by predicted class membership. We further displayed latent class-specific cumulative incidence functions for $\mathrm{BC}, \mathrm{CV}$ and other death by quartiles of the three markers. Finally, hazard ratios for $\mathrm{BC}, \mathrm{CV}$ and other death by levels of glucose, TG, and TC were estimated for each latent class according to the maximum-a-posteriori (MAP) likelihood, which took into account all three outcomes [19]. More details on the latent class survival analysis are available as Additional file 1.

Descriptive analysis and Cox proportional hazards model were performed with Statistical Analysis Software (SAS) release 9.3 (SAS Institute, Cary, NC) and $\mathrm{R}$

Table 1 Descriptive characteristics of study participants overall and by causes of death

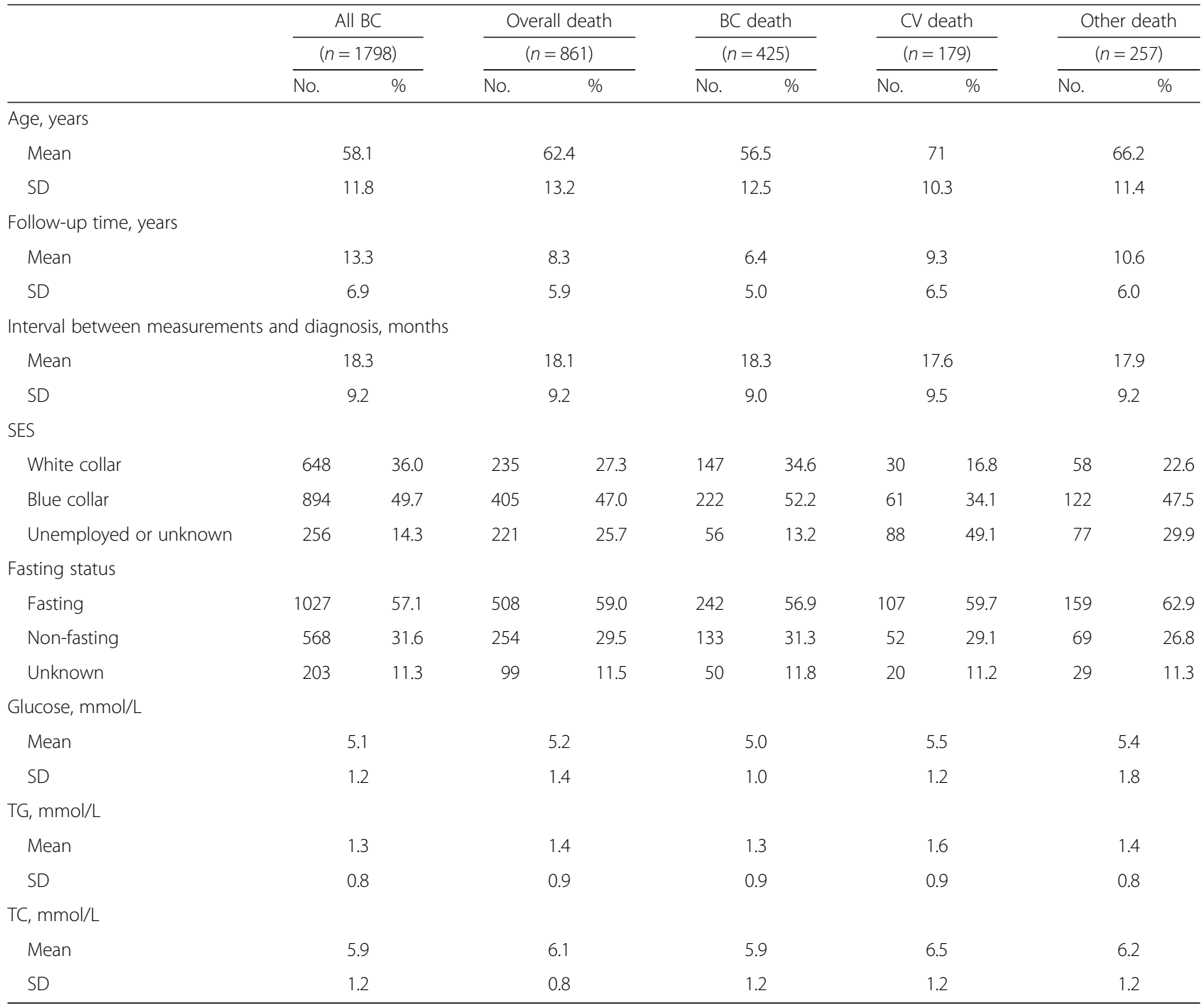


version 3.0.2 ( $\mathrm{R}$ Project for Statistical Computing, Vienna, Austria). Latent class proportional hazards model were performed with Advanced Survival Analysis software version 0.2.16 (A.C.C. Coolen, M. Rowley, M. Inoue, London, UK).

\section{Results}

At the end of follow up (mean: 13 years), a total of 861 (47.9\%) study participants were deceased. Among these women, 425 died from BC, 179 from CV disease, and 257 from other causes. The mean age of all participants was 58 at BC diagnosis. Levels of glucose, TG, and TC were highest in those dying from $\mathrm{CV}$ disease, whereas women who died from $\mathrm{BC}$ had lower levels of the three markers compared to all women dying during follow-up period (Table 1).
When conventional Cox proportional hazards regression was performed, no strong association was observed between glucose, TG, and TC and risk of dying from BC (Table 2). On the other hand, positive associations were observed between TG and CV death, as well as glucose and $\mathrm{CV}$ death. No association was observed for other causes of death. Proportions of deaths from each causes by quartiles of glucose, TG, TC was further displayed using the cumulative incidence functions. As shown in Fig. 1, the proportion of women dying from $\mathrm{CV}$ disease markedly increased with higher quartiles of the markers, whilst deaths from $\mathrm{BC}$ are less frequent with higher quartiles of the markers. This indicated $\mathrm{CV}$ death as a competing event.

Our next analysis extended the proportional hazards model to include latent class variables and assess primary and non-primary outcomes. Bayesian model

Table 2 Hazard ratios of death from BC, CV disease and other causes by levels of glucose, TG, and TC

\begin{tabular}{|c|c|c|c|c|c|c|c|c|c|c|}
\hline & \multirow{2}{*}{$\begin{array}{l}\text { No. of } \\
\text { subjects }\end{array}$} & \multicolumn{3}{|l|}{$\mathrm{BC}$ death } & \multicolumn{3}{|l|}{ CV death } & \multicolumn{3}{|l|}{ Other death } \\
\hline & & No. of events & $H R^{a}$ & $95 \% \mathrm{Cl}$ & No. of events & $H R^{a}$ & $95 \% \mathrm{Cl}$ & No. of events & $H R^{a}$ & $95 \% \mathrm{Cl}$ \\
\hline \multicolumn{11}{|l|}{ Glucose, $\mathrm{mmol} / \mathrm{L}^{\mathrm{b}}$} \\
\hline Continuous log & & & 0.96 & $0.58,1.59$ & & 2.48 & $1.24,4.96$ & & 2.09 & $1.16,3.76$ \\
\hline \multicolumn{11}{|l|}{ Quartiles } \\
\hline$<4.50$ & 393 & 98 & 1 & & 21 & 1 & & 45 & 1 & \\
\hline $4.50-4.90$ & 413 & 116 & 0.98 & $0.75,1.29$ & 36 & 1.27 & $0.74,2.19$ & 63 & 1.12 & $0.76,1.64$ \\
\hline $4.90-5.30$ & 363 & 96 & 0.95 & $0.72,1.27$ & 41 & 1.28 & $0.75,2.19$ & 50 & 0.87 & $0.58,1.30$ \\
\hline$\geq 5.30$ & 416 & 115 & 0.98 & $0.74,1.29$ & 80 & 1.67 & $1.02,2.73$ & 100 & 1.32 & $0.92,1.89$ \\
\hline$P_{\text {trend }}$ & & & 0.83 & & & 0.03 & & & 0.20 & \\
\hline \multicolumn{11}{|l|}{$\mathrm{TG}, \mathrm{mmol} / \mathrm{L}^{\mathrm{c}}$} \\
\hline Continuous log & & & 1.21 & $0.98,1.48$ & & 1.58 & $1.17,2.13$ & & 1.32 & $1.02,1.71$ \\
\hline \multicolumn{11}{|l|}{ Quartiles } \\
\hline$<0.70$ & 297 & 81 & 1 & & 12 & 1 & & 24 & 1 & \\
\hline $0.70-1.00$ & 491 & 102 & 0.77 & $0.57,1.04$ & 34 & 0.91 & $0.46,1.77$ & 56 & 0.96 & $0.59,1.57$ \\
\hline $1.00-1.60$ & 555 & 132 & 0.97 & $0.72,1.29$ & 52 & 1.10 & $0.58,2.08$ & 95 & 1.28 & $0.81,2.03$ \\
\hline$\geq 1.60$ & 455 & 110 & 1.05 & $0.76,1.45$ & 80 & 1.53 & $0.81,2.90$ & 83 & 1.22 & $0.75,1.98$ \\
\hline$P_{\text {trend }}$ & & & 0.35 & & & 0.01 & & & 0.16 & \\
\hline \multicolumn{11}{|l|}{$\mathrm{TC}, \mathrm{mmol} / \mathrm{L}^{\mathrm{d}}$} \\
\hline Continuous log & & & 0.72 & $0.40,1.28$ & & 2.04 & $0.83,5.04$ & & 0.67 & $0.32,1.42$ \\
\hline \multicolumn{11}{|l|}{ Quartiles } \\
\hline$<5.20$ & 443 & 119 & 1 & & 16 & 1 & & 38 & 1 & \\
\hline $5.20-5.80$ & 403 & 94 & 0.87 & $0.66,1.14$ & 37 & 1.52 & $0.83,2.76$ & 60 & 1.18 & $0.78,1.79$ \\
\hline $5.80-6.60$ & 470 & 102 & 0.79 & $0.60,1.04$ & 40 & 1.26 & $0.70,2.27$ & 75 & 1.06 & $0.72,1.58$ \\
\hline$\geq 6.60$ & 482 & 110 & 0.85 & $0.64,1.15$ & 85 & 1.74 & $0.99,3.04$ & 85 & 0.92 & $0.61,1.38$ \\
\hline$P_{\text {trend }}$ & & & 0.21 & & & 0.08 & & & 0.38 & \\
\hline
\end{tabular}

${ }^{\mathrm{a}}$ Adjusted for age at diagnosis, SES (white collar, blue collar, unemployed or unknown), fasting status (fasting, non-fasting, unknown), glucose (continuous), TG (continuous), and TC (continuous)

Not adjusted for ${ }^{\mathrm{b}}$ glucose, ${ }^{\mathrm{C}} \mathrm{TG},{ }^{\mathrm{d}} \mathrm{TC}$ 


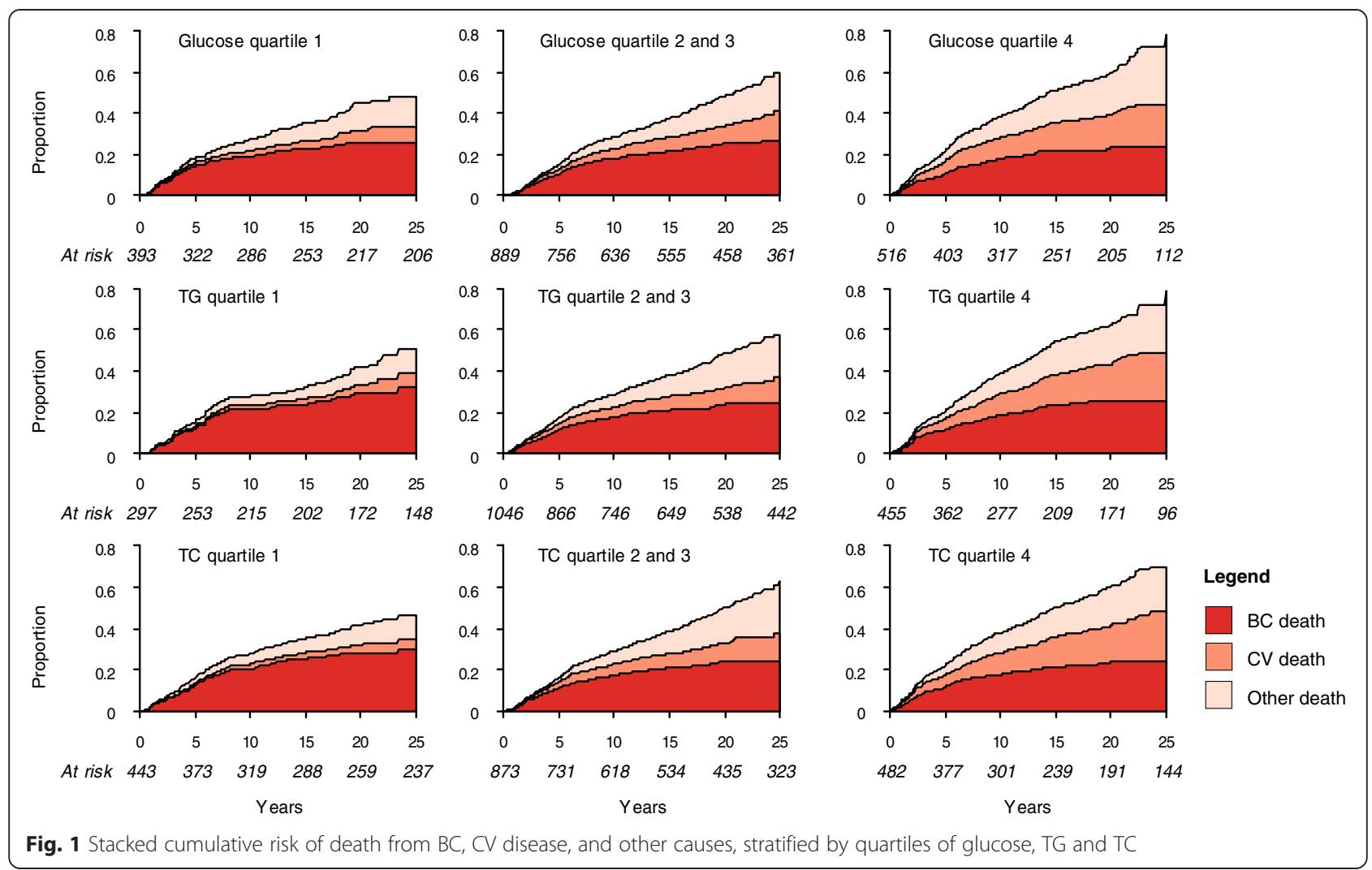

selection identified two latent classes in this study population. Retrospective analysis for class membership probability suggested that $81.5 \%$ women were more likely to be members of Class I, while the other $18.5 \%$ belonged to Class II. We further assessed baseline characteristics of study participants in relation to the most probable latent class they were assigned to. Younger average age was observed in Class I compared to Class II, and a difference in socio-economic status between classes was indicated (Table 3). With regards to clinical outcomes, no difference in proportions of women who died from BC was found between the two classes. However, statistically significantly higher overall mortality rate from $\mathrm{CV}$ disease and other causes were seen in Class II.

We further investigated difference in survivals between latent classes by displaying cumulative incidence functions for different causes of death by quartiles of glucose, TG, and TC (Fig. 2). Higher overall mortality was seen in Class II compared to Class I. In Class I, most patients died from BC, whereas in Class II, most died from other causes apart from $\mathrm{BC}$ and $\mathrm{CV}$ death. Increasing absolute numbers of deaths from $\mathrm{BC}, \mathrm{CV}$, and other causes were seen with higher levels of all three markers in Class I, although there was no marked difference in relative mortality rates between each cause of death. On the other hand, marked differences in relative proportions of women dying from the three different causes were seen across levels of markers in Class II. For instance, BC deaths were common amongst women in the lowest quartiles of glucose, TG, and TC, but contributed little to total deaths in those with highest levels of the markers. More women died from CV disease with higher $\mathrm{TC}$, and a similar association was seen between glucose and death from other causes. Finally, the risk of different causes of death was quantitatively assessed by obtaining class-specific hazard estimates. As seen in Table 4, logtransformed TG corresponded to an increased risk of dying from BC in Class I, with a hazard ratio of 1.87 (95\% CI: 1.01-3.45). No statistically significant associations with $\mathrm{BC}$ death were observed for other markers or among women in Class II. In agreement with classspecific cumulative incidence functions, women in Class II had a higher risk of $\mathrm{CV}$ death with higher $\mathrm{TC}$ and a higher risk of other death with higher glucose levels.

\section{Discussion}

We performed Cox regression and a latent class proportional hazards analysis to assess the association between prediagnostic markers of glucose and lipid metabolism and death from $\mathrm{BC}$ in female $\mathrm{BC}$ patients. The latter method accounted for $\mathrm{CV}$ death and other death as competing risks. With the conventional Cox 
Table 3 Characteristics of study participants and causes of death by predicted class membership

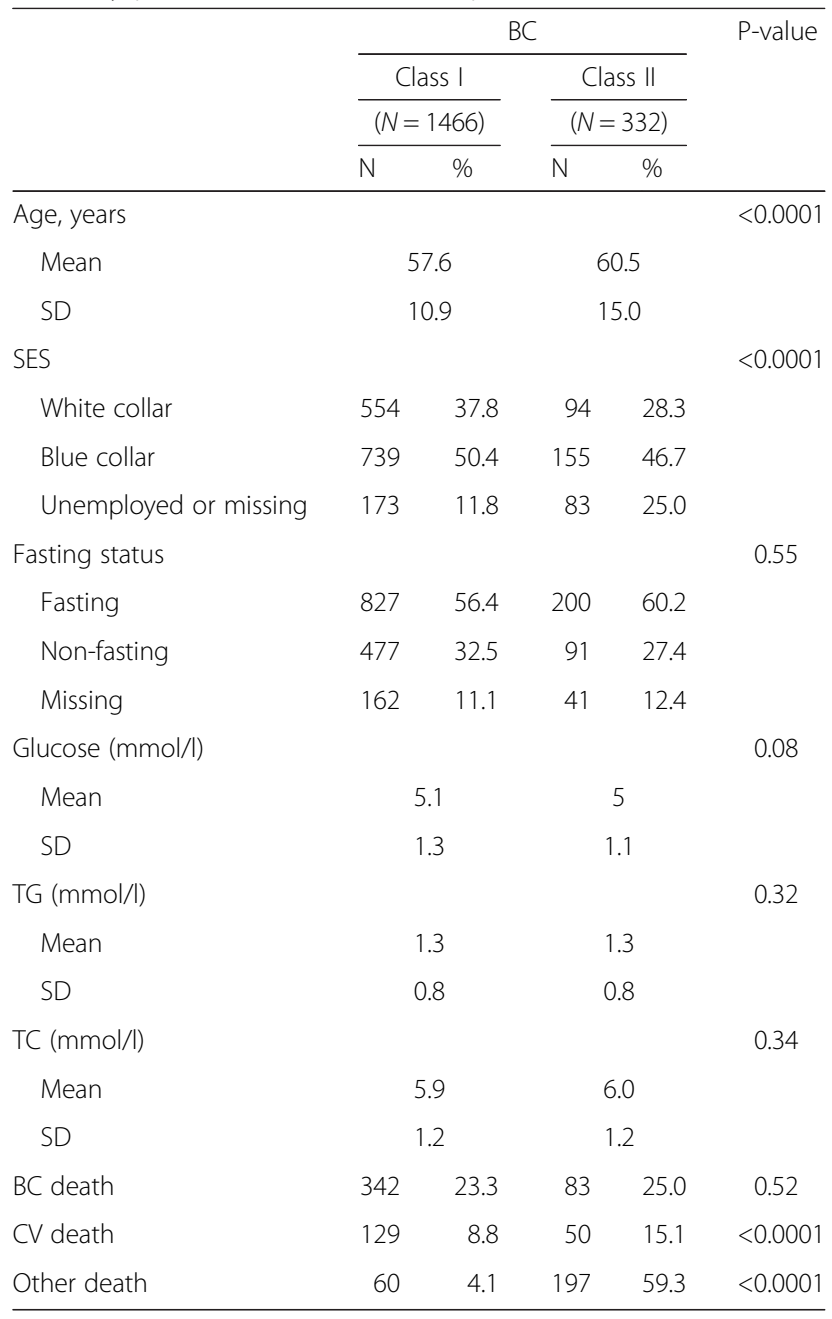

proportional hazards model, a lack of association was observed between the three markers and BC death. However, CV death was shown as a competing event. When latent class proportional hazards analysis were performed, we found two distinct latent classes within our cohort, reflecting different susceptibilities of dying from $\mathrm{BC}$ based on their baseline characteristics. Class I, comprising the majority of the study population, is associated with an increased risk of $\mathrm{BC}$ death following higher TG levels. Overall survival is worse in Class II, among which higher TC levels were associated with an increased risk CV death and higher glucose with risk of death from other causes. No association between the three markers and $\mathrm{BC}$ death was seen in Class II.

Metabolisms of glucose and lipid have been implicated in many chronic diseases. In the context of cancer, an array of evidence has linked increased $\mathrm{BC}$ incidence with aberrant levels of circulating glucose, TG and TC at baseline [20-22]. Abnormal levels of these markers are also associated with CV disease, which is the most common cause of death in general population [8,9]. This has also been demonstrated in our study, as both glucose and TG were associated with a higher risk of $\mathrm{CV}$ death, and the associations were stronger than those with BC death. Several biological mechanisms are suggested to underlie this common link, such as chronic inflammation and insulin resistance, which may drive atherogenesis, cellular proliferation and angiogenesis $[2,23,24]$. These shared metabolic pathways may thus result in a competing risks situation, where individuals with similar sets of risk factors are equally at risk of dying from both $\mathrm{BC}$ and $\mathrm{CV}$ disease. In this case, a heterogeneous association between glucose and lipid markers and BC death may be observed, which represents subpopulations or latent classes with different mortality risk profiles. However, this heterogeneity in survival data is not addressed by common analytical methods in cancer epidemiology.

Cox proportional hazards regression and latent classes proportional hazards model differ fundamentally in the assumptions made regarding risk correlations. In Cox, non-informative censoring is assumed, which leads to the assumption of independence or no correlation between event times when multiple events are observed. However, in the real-world clinical observation, such assumptions are rarely assessable and sometimes inaccurate. The latent class proportional hazards model allows for the presence of heterogeneity underlying any observed risk associations [16] and predicts optimal parameters based on the most probable substructure of the study population. In our study, this resulted in an optimal model with two latent classes. Overall survival was lower in Class II than Class I, which indicates the importance of taking into account risk associations when investigating biological markers in relation to cancer survival.

We found TG to be associated with early death from $\mathrm{BC}$ in Class I. This suggests an importance of lipid metabolism in disease progression in a relevant subset of $\mathrm{BC}$ patients, which warrants further mechanistic investigation. No statistically significant association with $\mathrm{BC}$ death was observed for glucose and TC, although among Class II they were associated with higher risks of dying from other causes and CV disease, respectively. Previous studies have reported a null association for TG and TC in relation to all-cause mortality [25] and BC-specific death [26], which is similar to our findings using Cox regression and in Class II as assessed by latent classes proportional hazards model. Likewise, a lack of association with overall death has been reported for glucose $[4,5]$. Although Class I comprised the majority of all women 


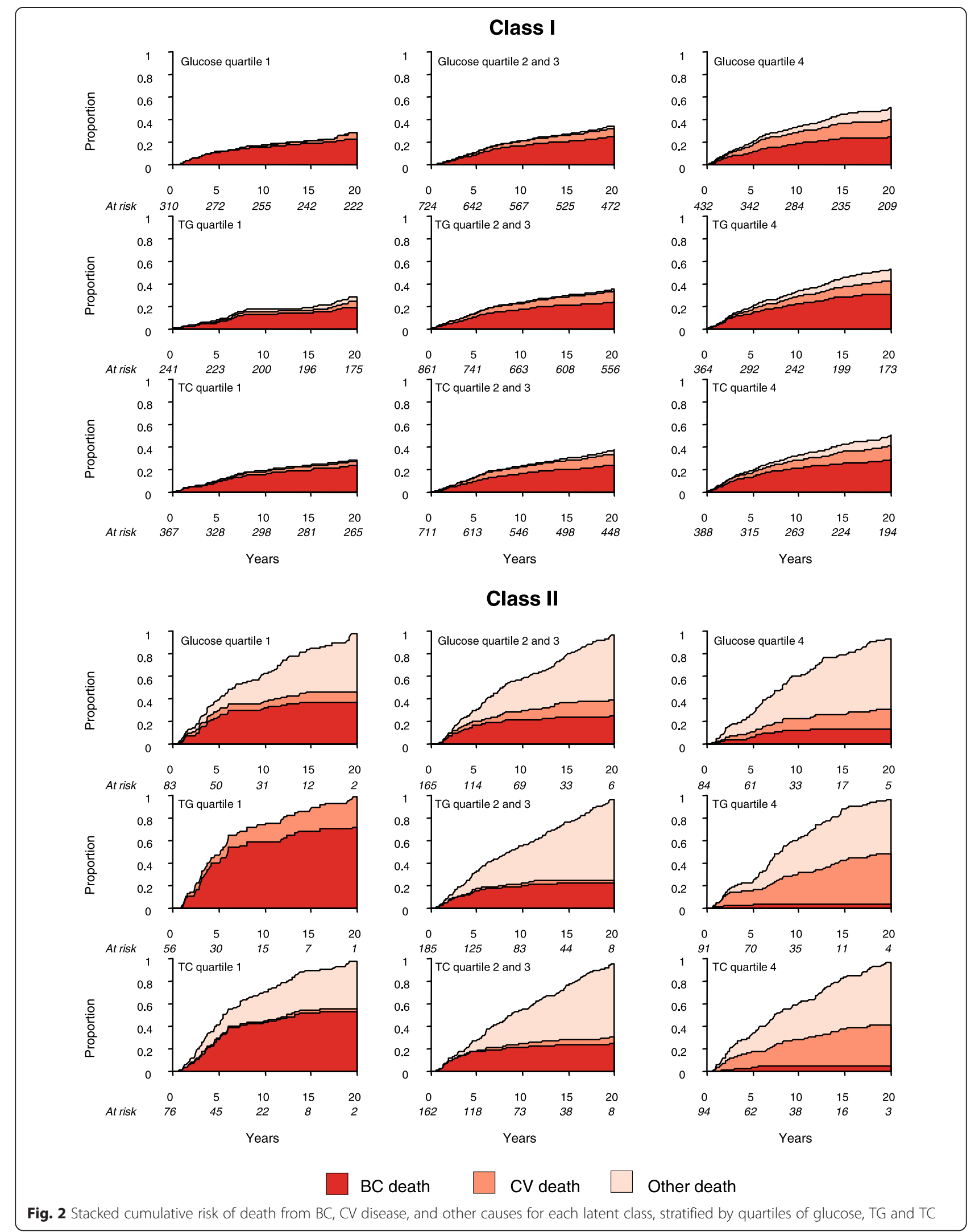


Table 4 Hazard ratios of death from BC, CV disease and other causes by levels of glucose, TG, and TC for each latent class

\begin{tabular}{|c|c|c|c|c|}
\hline & \multicolumn{2}{|c|}{ Class I } & \multicolumn{2}{|c|}{ Class II } \\
\hline & $\overline{\mathrm{HR}^{\mathrm{a}}}$ & $95 \% \mathrm{Cl}$ & $\overline{H R^{a}}$ & $95 \% \mathrm{Cl}$ \\
\hline \multicolumn{5}{|l|}{$\mathrm{BC}$ death } \\
\hline $\begin{array}{l}\text { Log } \\
\text { glucose }\end{array}$ & 1.09 & $0.73,1.63$ & 0.84 & $0.45,1.57$ \\
\hline $\log T G$ & 1.87 & $1.01,3.45$ & 0.91 & $0.50,1.68$ \\
\hline $\log T C$ & 0.84 & $0.49,1.45$ & 1.02 & $0.53,1.99$ \\
\hline \multicolumn{5}{|l|}{ CV death } \\
\hline $\begin{array}{l}\text { Log } \\
\text { glucose }\end{array}$ & 1.02 & $0.55,1.91$ & 1.46 & $0.97,2.20$ \\
\hline $\log T G$ & 7.68 & $2.45,24.02$ & 0.71 & $0.40,1.25$ \\
\hline $\log T C$ & 0.86 & $0.32,2.28$ & 2.07 & $1.16,3.69$ \\
\hline \multicolumn{5}{|c|}{ Other death } \\
\hline $\begin{array}{l}\text { Log } \\
\text { glucose }\end{array}$ & 0.73 & $0.50,1.05$ & 2.26 & $1.50,3.40$ \\
\hline $\log T G$ & 1.69 & $0.95,3.01$ & 1.40 & $0.74,2.64$ \\
\hline $\log T C$ & 1.20 & $0.65,2.24$ & 0.45 & $0.19,1.06$ \\
\hline
\end{tabular}

${ }^{a}$ All covariates were included in a single model and adjusted for age at diagnosis, SES (white collar, blue collar, unemployed or unknown) and fasting status (fasting, non-fasting, unknown)

studied, it is possible that the positive association between TG and Class I was diluted in the overall cohort, resulting in a weaker association. Therefore, it is important to consider cohort heterogeneity in assessing this relationship.

The strength of this study lies in the survival analysis method used to address competing risks, as well as the relatively large cohort with follow-up information for all participants (up to 25 years). The population in the AMORIS study was selected by analysing blood samples from health check-ups in non-hospitalised persons. However, any healthy cohort effect would not affect the internal validity of our study [11]. To our knowledge, this is the first observational study utilising latent class proportional hazards model to address disease-specific survival in $\mathrm{BC}$, taking into account $\mathrm{CV}$ death and other death as competing events. As shown in our study, the advantage of incorporating latent class analysis and multiple events in addition to proportional hazards regression is that it allows identification of subpopulations within the cohort and final survival or hazard estimates of the primary event. In other words, this method may offer a suitable approach when dealing with survival functions or hazard rates estimation in presence of competing risks. A limitation of our study was the lack of data representing older BC patients, which may partly explain the low proportion of Class II. There was no information available on tumour characteristics, BC susceptibility genes, and treatment or other metabolic and endocrine factors related to $\mathrm{BC}$ such as obesity and use of hormonal replacement therapy. Although residual associations with unobserved covariates were captured by our model through identification of latent classes, underlying characteristics of these different subgroups of $\mathrm{BC}$ patients may require further integration of other relevant markers or baseline information.

\section{Conclusion}

The present study showed a weak association between prediagnostic TG levels and $\mathrm{BC}$ death in the majority of women with BC. On the other hand, glucose and TC were strongly associated to mortality from causes apart from $\mathrm{BC}$ in the remaining patients, among which shorter overall survival was observed. Our study therefore demonstrated heterogeneity in the association between glucose, lipid markers, and BC survival when CV death and other death were taken into account as competing outcomes. This implies an involvement of perturbed lipid metabolism in $\mathrm{BC}$ progression and a complex interaction between baseline biological markers and co-morbidities in determining BC survival which warrants mechanistic investigations. Therefore, our findings highlight the importance of considering cohort heterogeneity when evaluating biological markers in relation to causespecific death.

\section{Additional file}

Additional file 1: Bayesian Survival Analysis with a latent class model. (DOC $37 \mathrm{~kb}$ )

\section{Competing interest}

The authors declare that they have no competing interests. Niklas Hammar is employed by the AstraZeneca, but the views expressed in the manuscript are his own and not those of AstraZeneca.

\section{Authors' contributions}

WW, MV, LH, HG and MVH conceived and designed the study. WW, LH, HG, $\mathrm{ML}, \mathrm{NH}, \mathrm{GW}, \mathrm{IJ}$, and MVH were responsible for data acquisition and quality control. WW, MV, MR, and ACC performed all data analysis. All authors interpreted study findings, prepared the manuscript and reviewed the final draft. All authors read and approved the final manuscript.

\section{Acknowledgement}

This work was supported by the National Institute for Health Research (NIHR) Biomedical Research Centre based at Guy's and St Thomas' NHS Foundation Trust and King's College London. The views expressed are those of the author(s) and not necessarily those of the NHS, the NIHR or the Department of Health. The authors also acknowledge support by the Swedish Cancer Society (Cancerfonden), the Gunnar and Ingmar Jungner Foundation for Laboratory Medicine, the Swedish Council for Working Life and Social Research, and Cancer Research UK.

\section{Author details}

${ }^{1}$ Cancer Epidemiology Group, Division of Cancer Studies, King's College London, London, UK. ${ }^{2}$ Institute for Mathematical and Molecular Biomedicine, King's College London, London, UK. ³Department of Surgical Sciences, Uppsala University Hospital, Uppsala, Sweden. ${ }^{4}$ Regional Cancer Centre, Uppsala, Sweden. ${ }^{5}$ Department of Epidemiology, Institute of Environmental Medicine, Karolinska Institutet, Stockholm, Sweden. ${ }^{6}$ Department of Medical Epidemiology and Biostatistics, Karolinska Institutet, Stockholm, Sweden. 
${ }^{7}$ AstraZeneca Sverige, Södertalje, Sweden. ${ }^{8}$ Department of Cardiovascular Epidemiology, Institute of Environmental Medicine, Karolinska Institutet, Stockholm, Sweden. ${ }^{9}$ Department of Medicine, Clinical Epidemiological Unit, Karolinska Institutet and CALAB Research, Stockholm, Sweden.

Received: 14 July 2015 Accepted: 12 November 2015 Published online: 17 November 2015

\section{References}

1. Simpson ER, Brown KA. Minireview: obesity and breast cancer: a tale of inflammation and dysregulated metabolism. Mol Endocrinol. 2013;27:715-25.

2. Belardi V, Gallagher EJ, Novosyadlyy R, LeRoith D. Insulin and IGFs in obesity-related breast cancer. J Mammary Gland Biol Neoplasia. 2013;18:277-89.

3. Villarreal-Garza C, Shaw-Dulin R, Lara-Medina F, Bacon L, Rivera D, Urzua L, et al. Impact of diabetes and hyperglycemia on survival in advanced breast cancer patients. Exp Diabetes Res. 2012;2012:732027.

4. Minicozzi P, Berrino F, Sebastiani F, Falcini F, Vattiato R, Cioccoloni F, et al. High fasting blood glucose and obesity significantly and independently increase risk of breast cancer death in hormone receptor-positive disease. Eur J Cancer. 2013;49:3881-8.

5. Contiero P, Berrino F, Tagliabue G, Mastroianni A, Di Mauro MG, Fabiano S, et al. Fasting blood glucose and long-term prognosis of non-metastatic breast cancer: a cohort study. Breast Cancer Res Treat. 2013;138:951-9.

6. Yu XQ, O'Connell DL, Gibberd RW, Coates AS, Armstrong BK. Trends in survival and excess risk of death after diagnosis of cancer in 1980-1996 in New South Wales, Australia. Int J Cancer. 2006;119:894-900.

7. Hortobagyi GN, de la Salazar Garza J, Pritchard K, Amadori D, Haidinger R, Hudis CA, et al. The global breast cancer burden: variations in epidemiology and survival. Clin Breast Cancer. 2005;6:391-401.

8. Seshasai SRK, Kaptoge S, Thompson A, Di Angelantonio E, Gao P, Sarwar N et al. Diabetes mellitus, fasting glucose, and risk of cause-specific death. N Engl J Med. 2011;364:829-41.

9. Di Angelantonio E, Sarwar N, Perry P, Kaptoge S, Ray KK, Thompson A, et al. Major lipids, apolipoproteins, and risk of vascular disease. JAMA. 2009;302:1993-2000.

10. Satagopan JM, Ben-Porat L, Berwick M, Robson M, Kutler D, Auerbach AD. A note on competing risks in survival data analysis. $\mathrm{Br} J$ Cancer. 2004:91:1229-35.

11. Holme I, Aastveit AH, Hammar N, Jungner I, Walldius G. Inflammatory markers, lipoprotein components and risk of major cardiovascular events in 65,005 men and women in the Apolipoprotein MOrtality RISk study (AMORIS). Atherosclerosis. 2010;213:299-305

12. Holme I, Aastveit AH, Jungner I, Walldius G. Relationships between lipoprotein components and risk of myocardial infarction: age, gender and short versus longer follow-up periods in the Apolipoprotein MOrtality RISk study (AMORIS). J Intern Med. 2008;264:30-8.

13. Ludvigsson JF, Otterblad-Olausson P, Pettersson BU, Ekbom A. The Swedish personal identity number: possibilities and pitfalls in healthcare and medical research. Eur J Epidemiol. 2009;24:659-67.

14. Van Hemelrijck M, Holmberg L, Garmo H, Hammar N, Walldius G, Binda E, et al. Association between levels of $C$-reactive protein and leukocytes and cancer: three repeated measurements in the Swedish AMORIS study. Cancer Epidemiol Biomarkers Prev. 2011;20:428-37.

15. Rabe-Hesketh S, Skrondal A. Classical latent variable models for medical research. Stat Methods Med Res. 2008;17:5-32.

16. Larsen K. Joint analysis of time-to-event and multiple binary indicators of latent classes. Biometrics. 2004;60:85-92.

17. Kenzik KM, Martin MY, Fouad MN, Pisu M. Health-related quality of life in lung cancer survivors: latent class and latent transition analysis. Cancer. 2015;121(9):1520-8. doi:10.1002/cncr.29232.

18. Zheng H, Tumin D, Qian Z. Obesity and mortality risk: new findings from body mass index trajectories. Am J Epidemiol. 2013;178:1591-9.

19. Mackay DJ. Information theory, inference, and learning algorithms. Cambridge: Cambridge University Press; 2003.

20. Kitahara CM, Berrington de González A, Freedman ND, Huxley R, Mok Y, Jee $\mathrm{SH}$, et al. Total cholesterol and cancer risk in a large prospective study in Korea. J Clin Oncol. 2011:29:1592-8.

21. His M, Zelek L, Deschasaux M, Pouchieu C, Kesse-Guyot E, Hercberg S, et al. Prospective associations between serum biomarkers of lipid metabolism and overall, breast and prostate cancer risk. Eur J Epidemiol. 2014;29:119-32.
22. Huang $Y$, Cai $X$, Qiu M, Chen $P$, Tang $H, H u$ Y, et al. Prediabetes and the risk of cancer: a meta-analysis. Diabetologia. 2014;57:2261-9.

23. Van Gaal LF, Mertens IL, De Block CE. Mechanisms linking obesity with cardiovascular disease. Nature. 2006:444:875-80.

24. Qian B-Z, Li J, Zhang H, Kitamura T, Zhang J, Campion LR, et al. CCL2 recruits inflammatory monocytes to facilitate breast-tumour metastasis. Nature. 2011;475:222-5.

25. Bahl M, Ennis M, Tannock IF, Hux JE, Pritchard Kl, Koo J, et al. Serum lipids and outcome of early-stage breast cancer: results of a prospective cohort study. Breast Cancer Res Treat. 2005;94:135-44.

26. Emaus A, Veierød MB, Tretli S, Finstad SE, Selmer R, Furberg A-S, et al. Metabolic profile, physical activity, and mortality in breast cancer patients. Breast Cancer Res Treat. 2010;121:651-60.

\section{Submit your next manuscript to BioMed Central and take full advantage of:}

- Convenient online submission

- Thorough peer review

- No space constraints or color figure charges

- Immediate publication on acceptance

- Inclusion in PubMed, CAS, Scopus and Google Scholar

- Research which is freely available for redistribution

Submit your manuscript at www.biomedcentral.com/submit 